\title{
UNIVERSAL AUTONOMOUS CONTROL AND MANAGEMENT SYSTEM FOR MULTIPURPOSE UNMANNED SURFACE VESSEL
}

\author{
Andrzej Stateczny \\ Gdańsk University of Technology, Poland \\ Marine Technology Ltd. Poland \\ Pawel Burdziakowski \\ Gdańsk University of Technology, Poland
}

\begin{abstract}
The paper presents design, structure and architecture of the Universal Autonomous Control and Management System (UACAMS) for multipurpose unmanned surface vessel. The system was designed, installed and implemented on the multipurpose platform - unmanned surface vessel named HydroDron. The platform is designed to execute hydrographic survey missions with multi-variant configuration of the survey system (payload?) including multi-beam echo sounder, sonar, LiDAR, automotive radar, photographic and spectral camera systems. The UACAMS designed to provide flexibility that enables to operate on the different kind of surface platform and different type of functional payload. The full system configuration provides all four level of autonomy starting from remotely controlled to full autonomous mission. Each level can be implemented and run depending on user specific requirements. The paper explains the differences between autonomous and automatic mission and shows how the autonomy is implemented into the presented system. The full hardware structural design as well as the software architecture are described. In order to confirm initial assumptions the applied system was tested during four-week sea trials and tuned for a selected vessel to confirm assumptions. In the project, also the original shore control station was designed, produced and tested for the vessel, including specific user controls and radio communication system. Conclusions sum up all crucial points of the design and system implementation process.
\end{abstract}

Keywords: unmanned surface vessel, autonomous, management, control, system

\section{INTRODUCTION}

A proper control of multipurpose unmanned surface vessel, or generally unmanned vehicle, needs an advanced and sophisticated management system. The universal management system designed for multipurpose unmanned vessel, should have ability to deal with different type of functional payload, different tasks and situation. Moreover, general-purpose (universal) systems should have ability to be tailored to manoeuvring parameters of different vessels.

Among Unmanned Surface Vehicle (USV) solutions available on the market, single-hull platforms and catamarans can be indicated. During subsequent visits to Oceanology International Conference held in London in 2012, 2014, 2016 and 2018 it was easily seen a significant increase in number of presented solutions for USV, just as it happens in the market of unmanned aircraft. The USVs are produced in a range from small "pocket” platforms that can be carried in the trunk of the car to larger units of a nearly seagoing range and usually equipped with Multi-Beam Echo Sounder (MBES). see Fig. 1-11 (the photos taken by A. Stateczny during Oceanology International Conferences, London) 


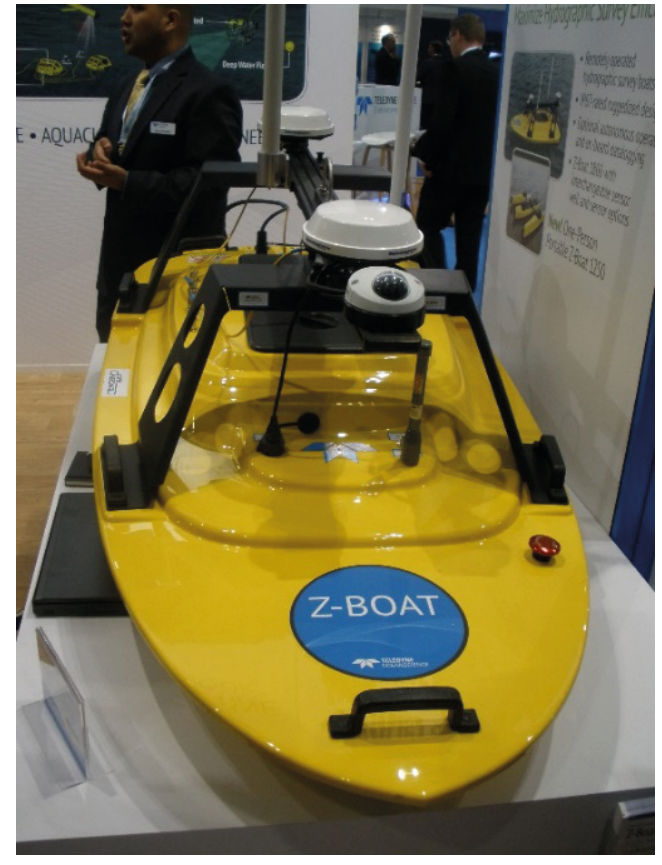

Fig. 1. Z-boat



Fig. 2. Ocean Nautical Innovations

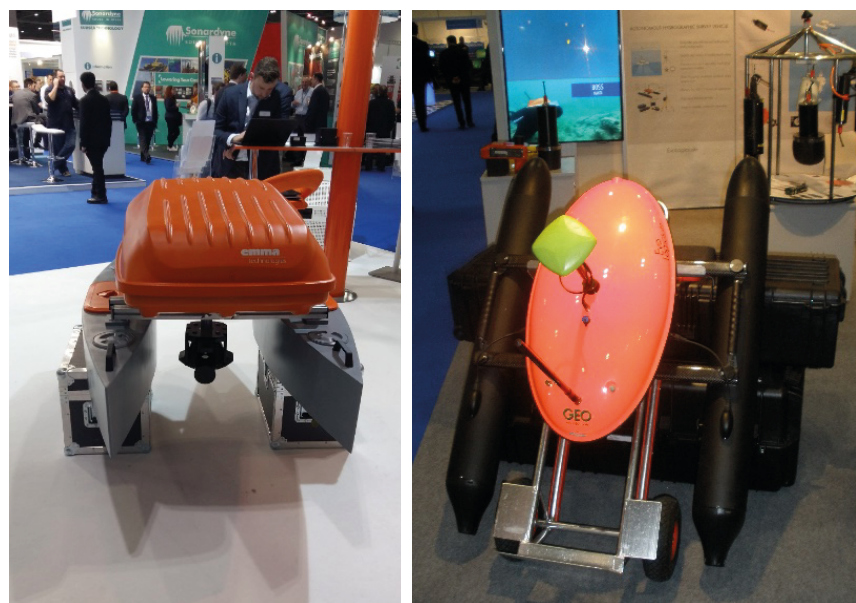

Fig. 4. HyCOPS (on the left) and Sonobot (on the right)

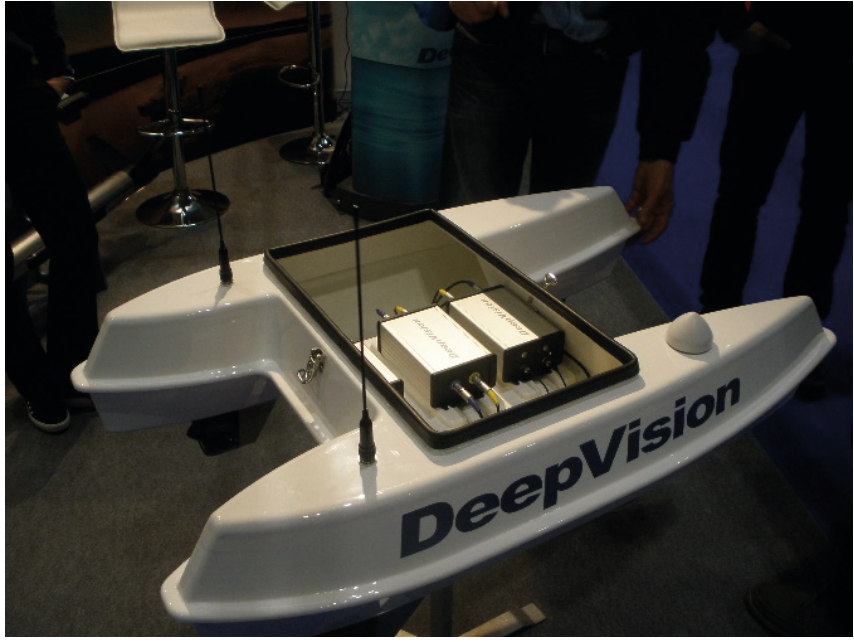

Fig. 5. DeepVision

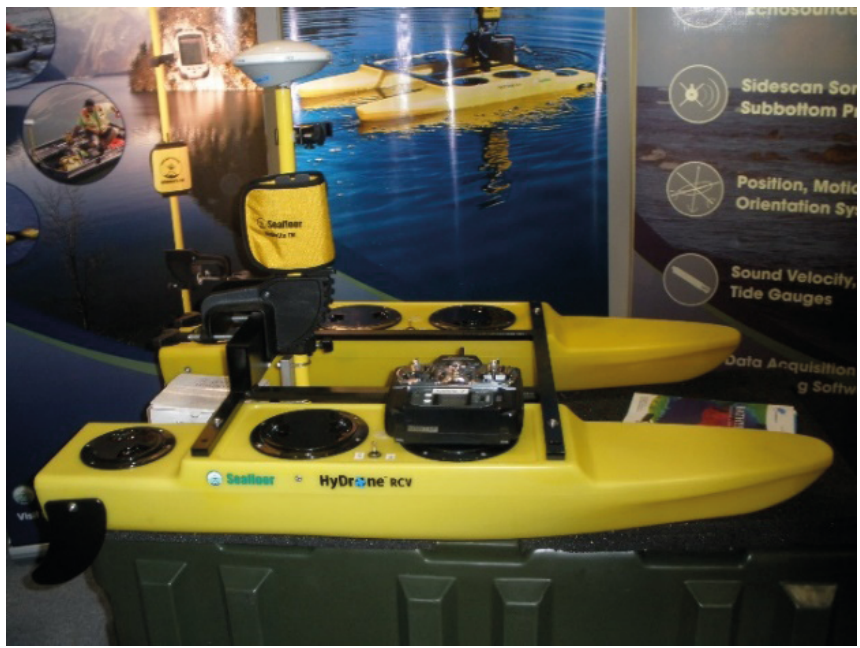

Fig. 6. HyDrone

The HyDrone was introduced to Polish market by Gdynia Maritime University [21,22].
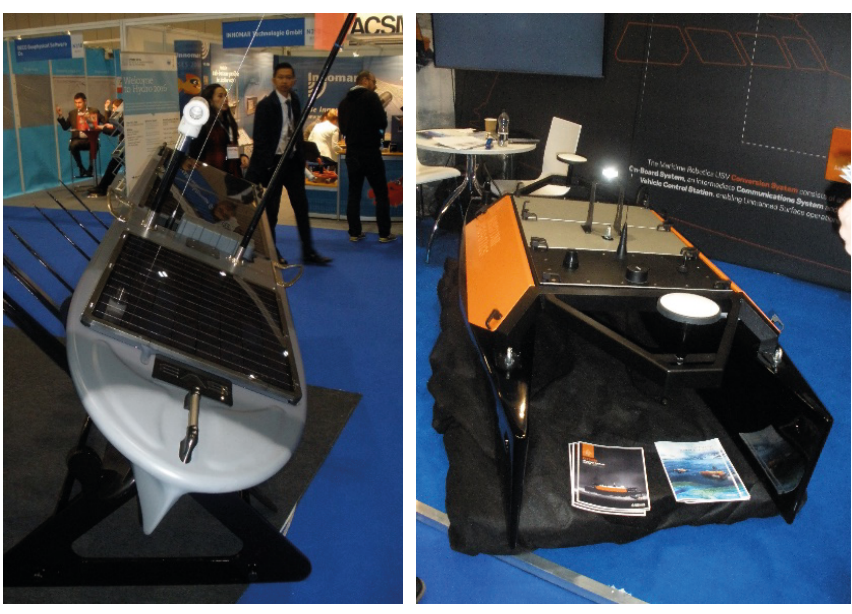

Fig. 7. Solar boat (left)and Marine Robotics (on the right) 


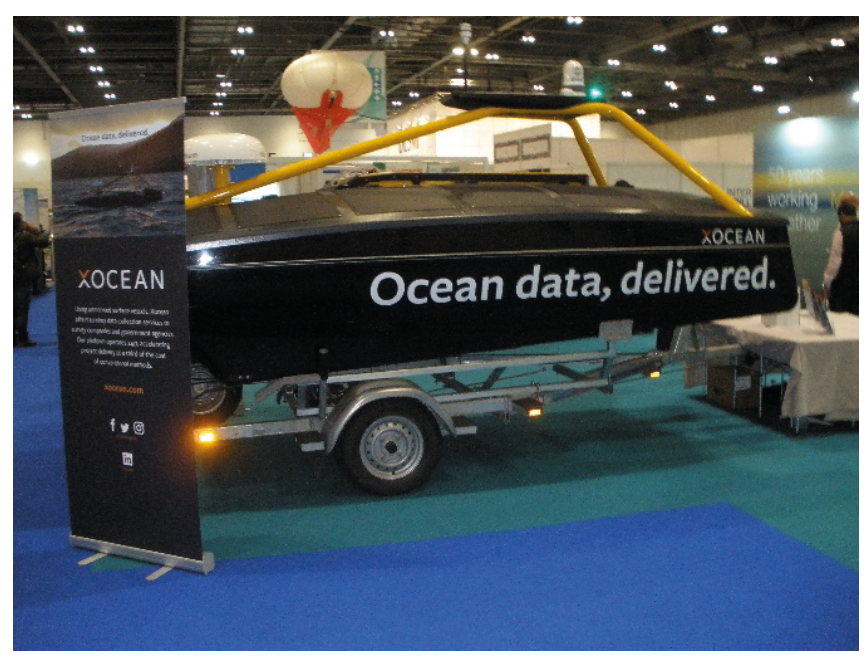

Fig. 8. XOCEAN

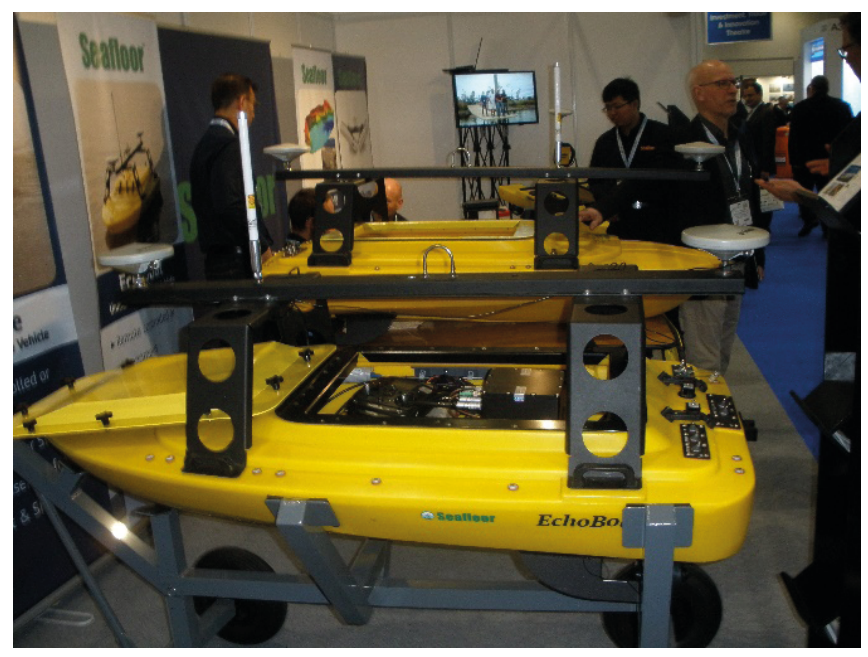

Fig. 9. Echoboat

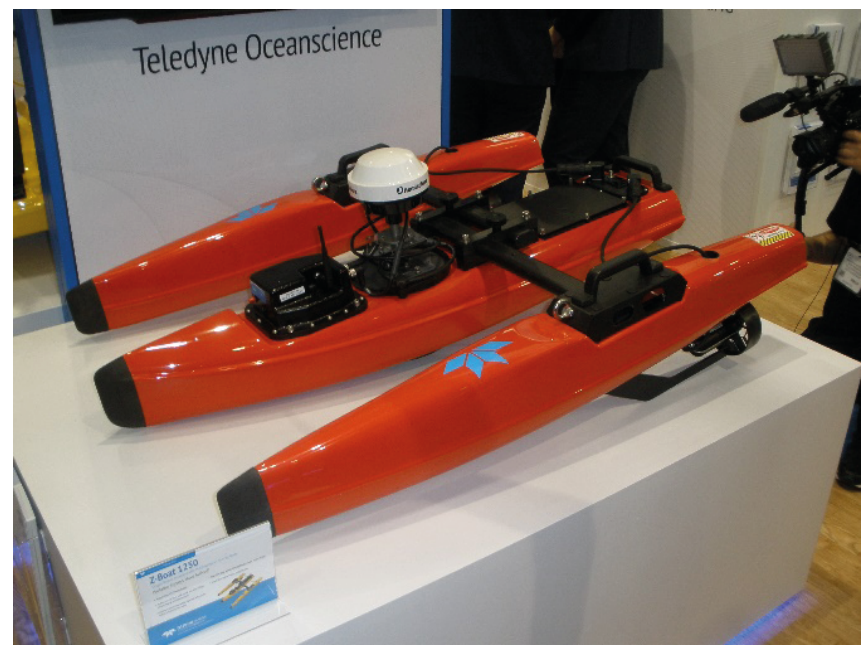

Fig. 10. Teledyne Oceanscience

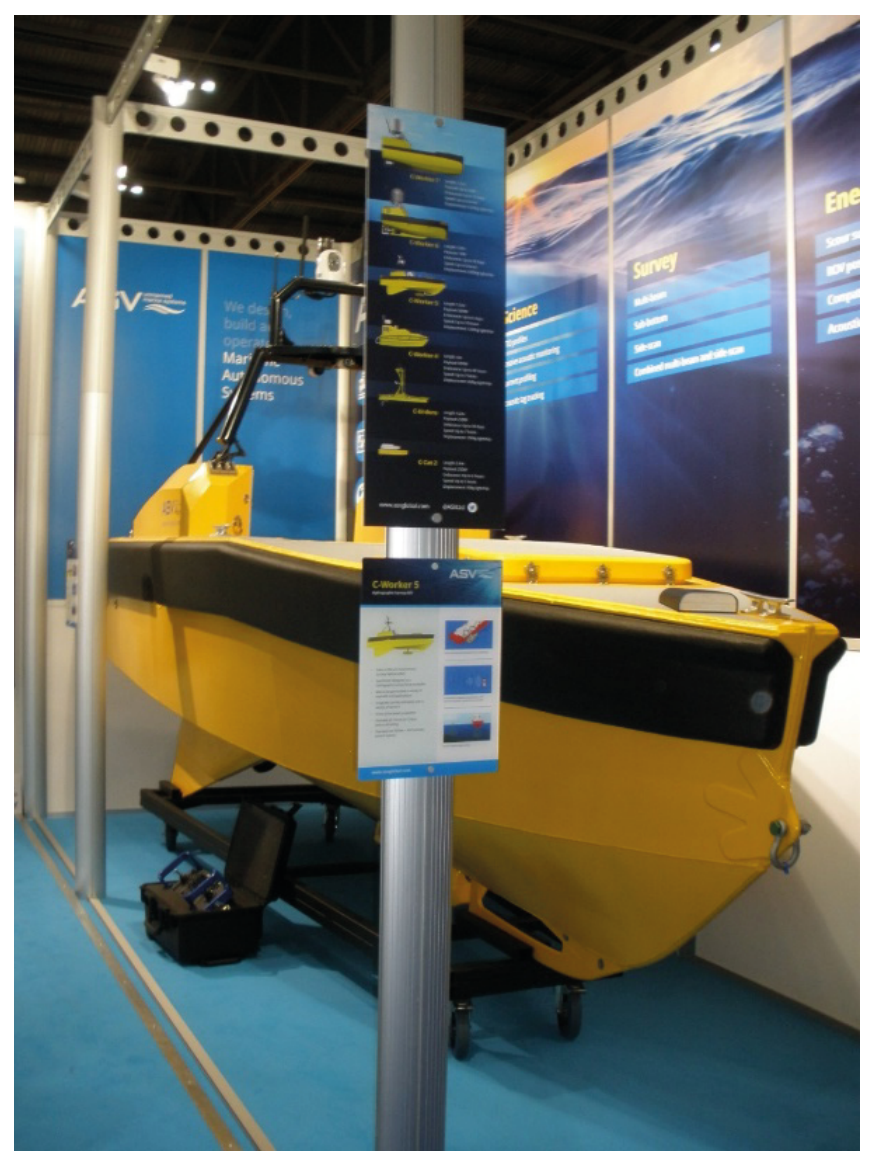

Fig. 11. C-Worker - whole set solutions

The presented overview of USVs offered on the markets indicates that many companies undertake the studies related to the USV development for hydrographic measurements in shallow waters. It is a strongly growing market which clearly could be seen during subsequent visits to the Oceanology International Conference in London.

In recent years in Poland, there were developed unmanned floating platforms such as UPP-1E platform implemented by the Department of Environmental Engineering, Cracow University of Technology, and Edredon platform - by the Polish Naval Academy. Currently there are not known any attempts to developing ASV construction besides HydroDron constructed by Marine Technology Ltd.

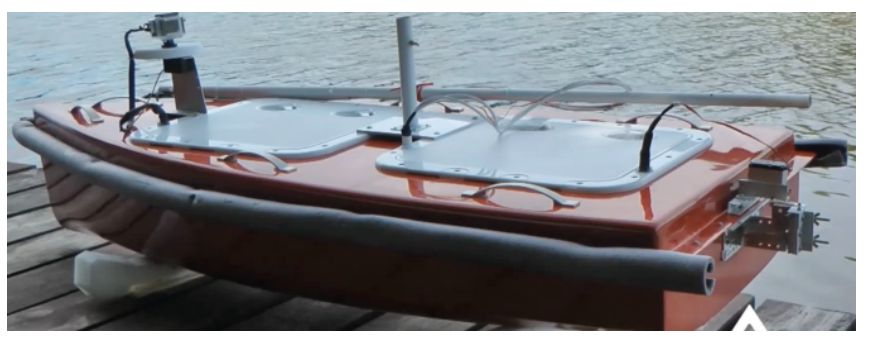

Fig. 12. UPP-1E universal measuring floating platform 
The UPP-1E platform is intended for bathymetric measuring but in terms of technology cannot be compared with the Edredon. The differences result from the construction parameters, installation of different sensors and their control systems. The Edredon was built on the basis of RIB hybrid boats (Rigid - Inflatable Boat) in 2009-2011.

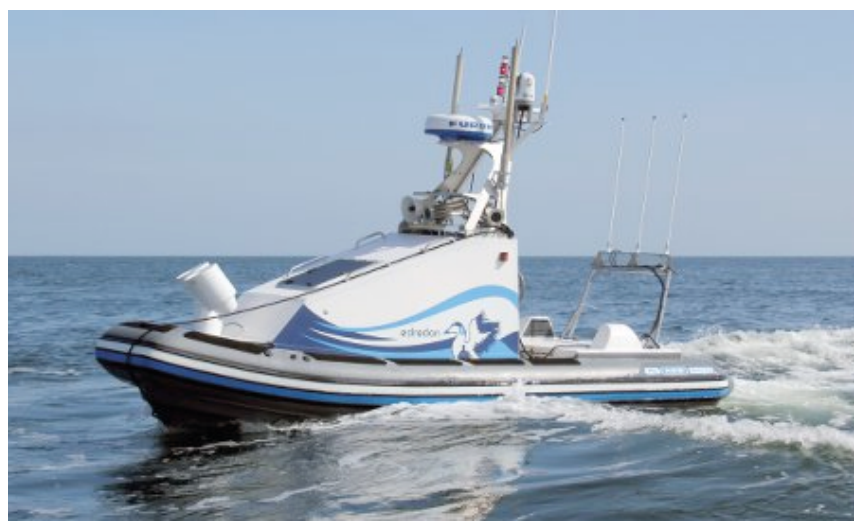

Fig. 13. The Edredon during the tests

Both the platforms represent a significant intellectual contribution to the development of design ideas for unmanned floating platforms in Poland.

The new platform named HydroDron, which is under construction by Marine Technology Ltd have significant advantages distinguishing it from the aforementioned solutions. Components installed on the platform are characterized by significantly improved characteristics in comparison with the aforementioned ones.

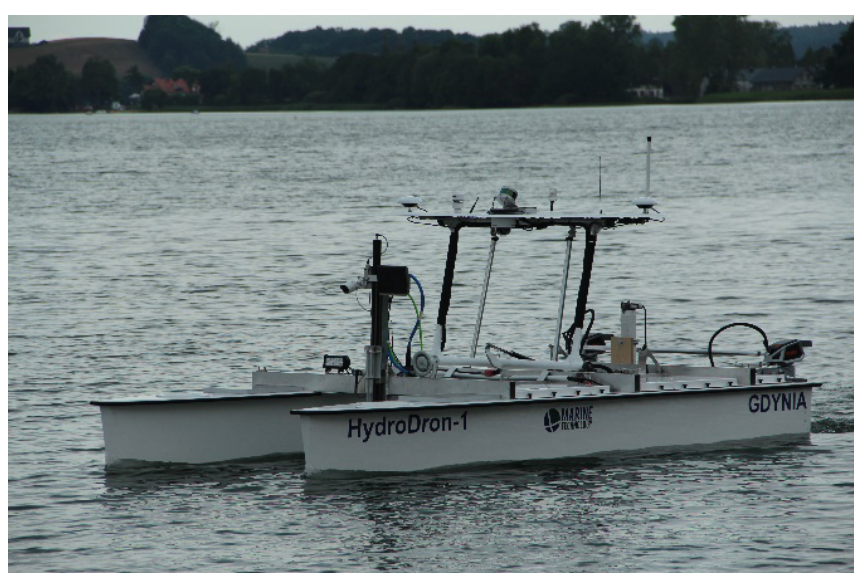

Fig. 14. The HydroDron during the tests

The HydroDron for hydrographic measurements is the product significantly ahead of previously available solutions on the Polish market and foreign markets. This applies not only to its technical parameters and characteristics, but also a purpose of using. It is assumed that the vehicle is able to operate in shallow waters where due to the presence of underwater obstacles at shallow depths a high precision navigation is required. The platform have a very high computational potential. The Marine Technology company has considerable experience in developing solutions for the maritime economy with a high level of intelligence. This also applies to collision avoidance systems based on solutions which use artificial intelligence and multisensory data fusion. Artificial intelligence is frequently implemented for autonomous navigation $[1,14,20]$ or for optimal ship's trajectory control [16-17]. The combination of multi-task platform with intelligent maneuvering will allow to offer an innovative product in terms of autonomy characterized by novelty on an international scale. Its floating structure allows to perform tasks in an autonomous manner, while ensuring a high accuracy required e.g. in dredging work. It is a technological challenge that the HydroDron design team set itself as the goal. This challenge forces to apply an innovative method of connecting accuracy of platform positioning with that of hydrographic measurements. In this regard the Marine Technology company uses the existing experience in the hydrographic work for developing an innovative product.

\section{UNMANNED VESSEL SYSTEM DESIGN}

Unmanned surface vehicle (USV) can be defined as a generic vessel designed to operate without human operator on board. The definition can be referred to any unmanned vehicle, including aerial, ground and underwater, and can be used for any kind of vehicle able to operate without human on board [2]. The typical unmanned vehicle is a part of the unmanned system, in the presented case - Unmanned Vessel System (UVS). While the USV is referred only to the surface platform, the whole unmanned vessel system includes a vehicle, shore control station (SCS), communication and control link (C2) and logistic module (Fig.15).

The basic USV modules are placed on board unmanned platform (vessel) and there are a mission control module, autonomous control module, sensors module, effectors and payload.

The mission control module consists of hardware and software responsible for navigation, orientation and automatic algorithms calculation. This module is fed with data from sensors module, operator's remote commands and autonomous module, and, based on currently established command priorities, it generates appropriate executive commands to the effectors module. Depending on the commands priorities (currently - ordered level of mission) the control module (Fig.16) directs the platform to a desired position and orientation.

The autonomous control module consists of hardware and software responsible for high level autonomous algorithms calculation including dynamic and adaptive path planning, collision avoidance and multisensory fusion. The module is fed with data from sensors module. Based on the sensor data and their fusion, the module continuously analyzes the overall situation and calculates required action. As in the navigation module, depending on the currently ordered level of autonomy, the autonomous control module sends the commands to the mission control module and to the 
operator display. In fully autonomous mode, the mission control module's commands are overwritten by the calculated autonomous action. In semi-autonomous mode, the required action is presented onto the operator display as a navigation advice. In this case, the operator can use this information and manually direct the unit to the required action, acknowledge the required action and make the system to automatically perform it, or ignore the suggested action.

The sensors module, a part of the unmanned vessel system, consists of all sensor installed on board the unit.

The sensors module includes all environment, anticollision, electrical, diagnostic, hydrographic (payload) and navigation sensors. Sensor information can be shared within the system or used only by a specific system.

The supportive systems contain all necessary systems, outside the UVS, which are required to conducting USV mission, such as the global navigation satellite system (GNSS), network for real-time-kinematic (RTK) positioning reference stations, etc.

The effector is defined as a device used to produce a desired change in an object in response to input. In the presented case, the propulsion and ruder system to drive the platform is the effector, as well as all other actuators and servos mounted on board the platform to drive remaining mechanical devices. The input signal to the effectors is generated by the mission control module as a result of automatic control or autonomous control calculations.

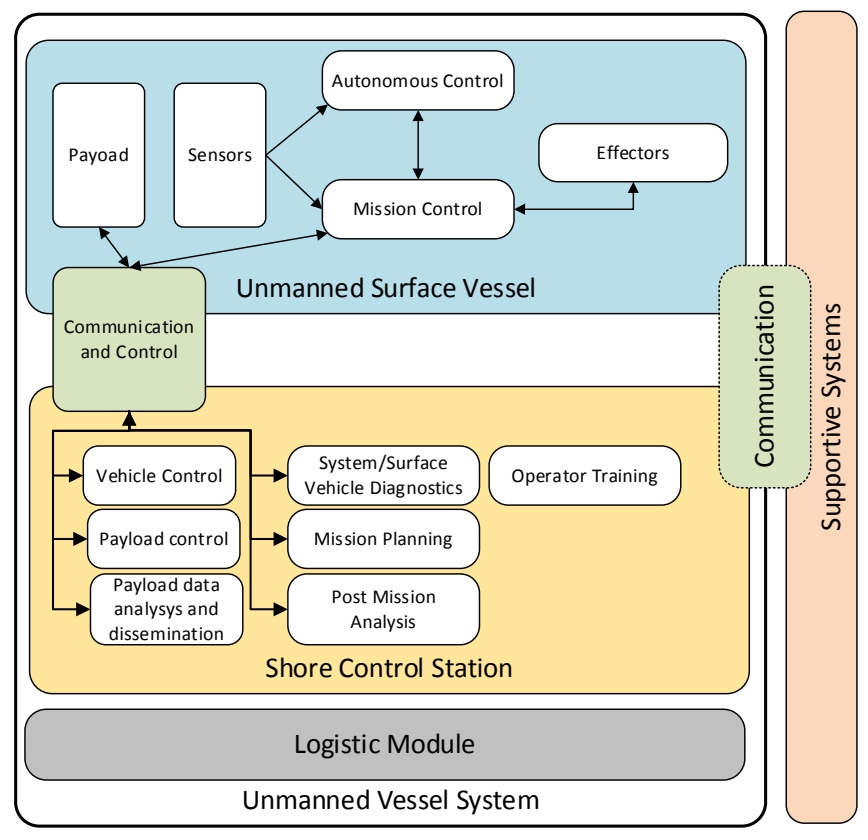

Fig. 15. Unmanned Vessel System modules (acc. these authors)

Depending on main purpose and tasks of a USV, its payload can be differently configured and equipped. In the presented case the USV is designed to carry out hydrographic survey missions in areas of ports, embankments, anchorages, bays and lakes, rivers and other restricted water areas and its main payload equipment includes professional bathymetric systems [23].
The shore control station (SCS) can be defined as stationary or transportable devices intended to monitor, command and control the unmanned vessel. The crucial functionalities of the shore control station are [2]: vehicle control - a capability to effectively control the USV during its mission, payload control - ability to operate payload sensors from the shore, mission planning - functionality that aids USV's operator in planning the mission providing required knowledge inputs concerning capabilities and USV's limitations, payload data analysis and dissemination - capability to disseminate the data from payload to eventual users, system/surface vehicle diagnostics - automatic test facility for USV and SCS effective maintenance and deployment, operator training - facility to train the surface vehicle operator in handling the vessel, practicing mission plans and emergency procedures, postmission analysis - capability to store mission data and payload data and to analyze it after the flight mission.

\section{AUTONOMOUS VERSUS AUTOMATIC MODE}

Within autonomous vehicles group, the term "autonomous" is sometimes incorrectly used for defining fully automatic unmanned systems. As an adjective "automatic" means capable to operate without external control or intervention. It means that the vehicle is capable of following a programmed scenario or situation and all its reactions are already assigned in advance and implemented into vehicle's processor (mission controller) by the developer. "Autonomous" stands for selfgoverning, intelligent, sentient, self-aware, thinking, feeling, governing independently, and in application to a vehicle or robot means that it not only performs one or more task automatically, but is able to perform decision-making process independently, i.e. without operator's inputs and, the most important, in a complex situation not previously assigned. The autonomous decision is made on the basis of previous knowledge and experience collected by the vehicle. The autonomous vehicles are able to act in complicated, real-world environment, while automatic vehicles are not. Automatic vehicle will always need a human aid to realize decision -making process. The remotely controlled vehicles base on human control inputs, and are not able to perform any tasks without such control. It is important to notice, that for safety reason, any autonomous or automatic vehicles are to be equipped with remote control. The remote control should be considered as a safety pilot in case of any unpredictable behaviour of machine. The semi-autonomous vehicles perform some tasks independently, and some are to be acknowledged by the human operator.

According to the range of autonomy, unmanned vehicles can be divided into several levels (Fig.16):

Level 1: remote control mode (manual mode); on this level the unit is able to perform commands received form operators station (remote control). This is the lowest level in which any navigation or anti-collision system or sensors are engaged. It can be used for manual docking operation or in 
case of malfunction of any sensor. Remote control (manual control) is strongly recommended for every autonomous system in order to have the possibility to overwrite the higher level commands - in this case remote control acts as a safety pilot. Every command generated by a mission control module or autonomous module can be overwritten in any time by a operator's manual command.

Level 2: automatic mode (steering, auto, return to home, smart return modes); on this level the unit uses internal navigation sensors and navigation algorithms to direct the unit. This level requires PID controllers (proportionalintegral-derivative controller) to be tuned and for navigation sensors readings. The unit uses a compass (9 magnetometers), GNSS receiver to perform basic commands (steering mode), a mission plan to perform automatic mission (auto mode), home location (dock location) to perform return to home port (dock) task, and saving the path to perform smart return to home mission.

Level 3 : semi-autonomous mode- covering full level 2 functionality (automatic) with autonomous system advice. All anti-collision additional sensors are active (i.e. two depth echo sounders, omnidirectional lidar, front and back sector lidars, front anti-collision radar) and anti-collision module calculates the manoeuvres, analyzes situation and generates an advice. The navigation advice is displayed on the operator panel. Operator is able to acknowledge or reject the manoeuvre. This mode is perfect for autonomous system tuning and testing. The autonomous neural network support learning phase can be monitored and controlled. Laser scanner for autonomous navigation is quite popular and was presented in the literature $[3,5,7,8,9,15,18,24]$. Radar sensor in not so popular like laser sensor but also was presented in some papers $[4,10]$.

Level 4: autonomous mode - in which all navigation and anti-collision systems and sensors are active. The autonomous module directs its platform without operator attention, according to the mission plan and actual situation. In this mode remote controller is used only for safety reason.

In the case of the presented project, a system including software, hardware and overall architecture is developed to such extent as to be able to cover all autonomy levels, including its main assumptions, beginning form remote control and ending on autonomous task realization. All four autonomy levels have been achieved on every development stage of the project. It was assumed, that all hardware configurations have to be prepared to cover all autonomous requirements and be open to integration of new sensors in the future.

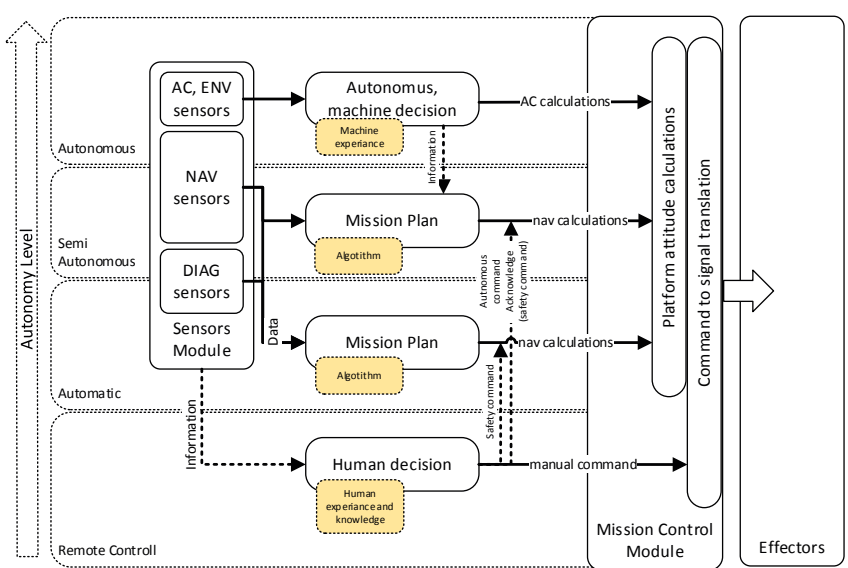

Fig. 16. Autonomy levels and minimally required modules (acc. these authors)

\section{MULTIPURPOSE DESIGN CAPABILITIES}

Multipurpose capabilities are achieved basing on a modular and open hardware and software architecture. All used algorithms, after proper tuning process, are able to operate on different hardware and direct any surface vessel different in type and size.

The automatic (mission control) algorithms are based on PID controller, what according to [6] was proved to be a sufficient method to control speed and course of surface vessel. The PID controller continuously attempts to minimize the error over time by adjustment of the control variable. The error value function is a difference between a desired set point and measured process variable PID controller function can be expressed as follows:

$$
u(t)=K_{p} e(t)+\frac{1}{T_{i}} \int_{0}^{t} e(t) d t+T_{d} \frac{d e(t)}{d t}
$$

where $K_{p}, K_{i}$ and $K_{d}$ are non-negative quantities which denote the coefficients for the proportional, integral, and derivative terms, respectively. Depending on the vessel type, size and maneuvering parameters the PID coefficients are tuned in live tuning process. PID desired response and PID achieved response are monitored and displayed and the coefficients can be adjusted to achieve an appropriate object's response and stabilization of its parameters (course and speed).

The high level of calculation relies on Robot Operating System (ROS). The ROS provides libraries, tools, hardware abstraction, device drivers, visualizers, message-passing and package management necessary to form robot applications. The ROS is used for calculation of anti-collision sensor data. It is a flexible framework for writing any robot software. This enables to simplify the task of creating complex and robust vehicle behaviour. The ROS enables to implement new sensors and change the sensors configuration without changing main anti-collision method. 


\section{HARDWARE ARCHITECTURE}

Hardware architecture and protocol integration scheme (Fig.17) is open to modifications, hardware changes and new sensors integration. The concept divides the hardware into a low- level and high- level hardware. The low- level hardware is responsible for automatic calculation, including safety actions. The low-level simple driver and controllers provide reliability to the system basic functions. The mission control module is based on 32bit STM32F427 Cortex-M4F core with FPU (floating-point unit), $168 \mathrm{MHz} / 252 \mathrm{MIPS}, 256 \mathrm{~KB}$ RAM, 2 MB Flash (fully accessible) and 32bit STM32F103 failsafe co-processor (the additional processor only for trigger safety manoeuvre and system actions). The mission control module is supplied with redundant power inputs and automatic failover system (3 different power source).

The anti-collision module and payload storage module are assigned to the high level hardware and created based on two fan-less industrial commuters (Aplex ACS-2332). Potential malfunction of the high level hardware does not affect the low-level hardware. Each commuter is equipped with significant number of I/O ports $(6 \mathrm{x}$ USB, 4 serial RS-232/422/485, 3 display interfaces, GPIO and 4 x GbE LAN interfaces), what enables to connect a number of different sensors directly to the machine.

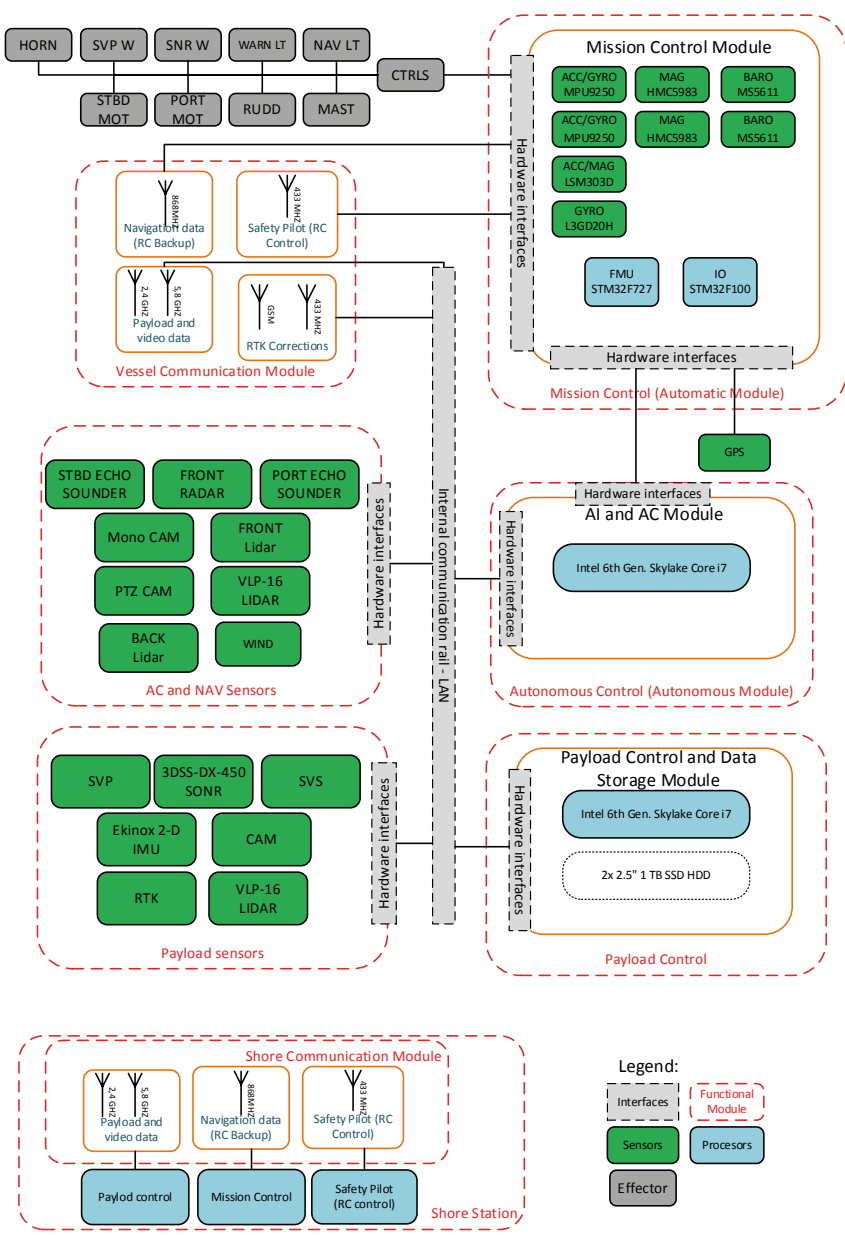

Fig. 17. Hardware architecture (acc. these authors)
NMEA (National Marine Electronics Association) communication based sensors are connected to the vessel internal network by means of the NMEA to LAN converter (MiniPlex-3E), and information is sent over the local network to all subscribers. All Ethernet- based sensors are integrated with internal LAN by means of two configurable switches (MikroTik). This concept allows to share all sensors information (including payload bathymetry sensors) within the system. The shared information is used by the anticollision module and adaptive planning navigation system and recorded in the storage module for further analyses. Data from all sensors are broadcasted to the local network and can be used by the system modules as required. The vessel internal network is connected by means of wireless distribution system (WDS) to the shore station local network. Such connection enables to share sensor information to the shore station modules.

\section{SOFTWARE ARCHITECTURE}

The software architecture provides full sensor integration capabilities and is based on open- source application and software (except payload control software) (Fig.18).

The mission control module is based on NuttX operating system ( NuttX is a real-time operating system (RTOS) with an emphasis put on standards compliance and small footprint, scalable from 8-bit to 32-bit microcontroller environments).

The collision avoidance module runs Linux Ubuntu software with ROS framework of modules having their own AI (artificial intelligence) and path-finding algorithms [13]. Different software implementations operate within different nodes.

In the nodes particular computations are performed. The presented USV control system comprises many nodes (for example, one node controls a laser range-finder, one node controls the radar computation, one performs path planning, one node provides a graphical view of the system, etc). The nodes produce topics - messages which are routed through a transport system with publish / subscribe semantics. A node sends out a message by publishing it to a given topic. A node that is interested in a certain kind of data will subscribe for an appropriate topic. Very important problem is multisensory data fusion; this issue was undertaken by several authors [11-13].

Payload control module runs Windows 10 and Hypack $^{\circledR}$ software for full bathymetry payload control and hydrographic adaptive mission planning. The Hypack ${ }^{\otimes}$ provides hydrographic surveying packages with all the tools needed to design survey, collect data, process it, reduce it, and generate final products. The payload software is independent form other software used on the vessel. Additionally, the Hypack can creates adaptive survey grid based on the already measured data. The generated track are sent through internal LAN to the mission control module and the vessel can adjust current survey grid to the already collected bathymetric data. 


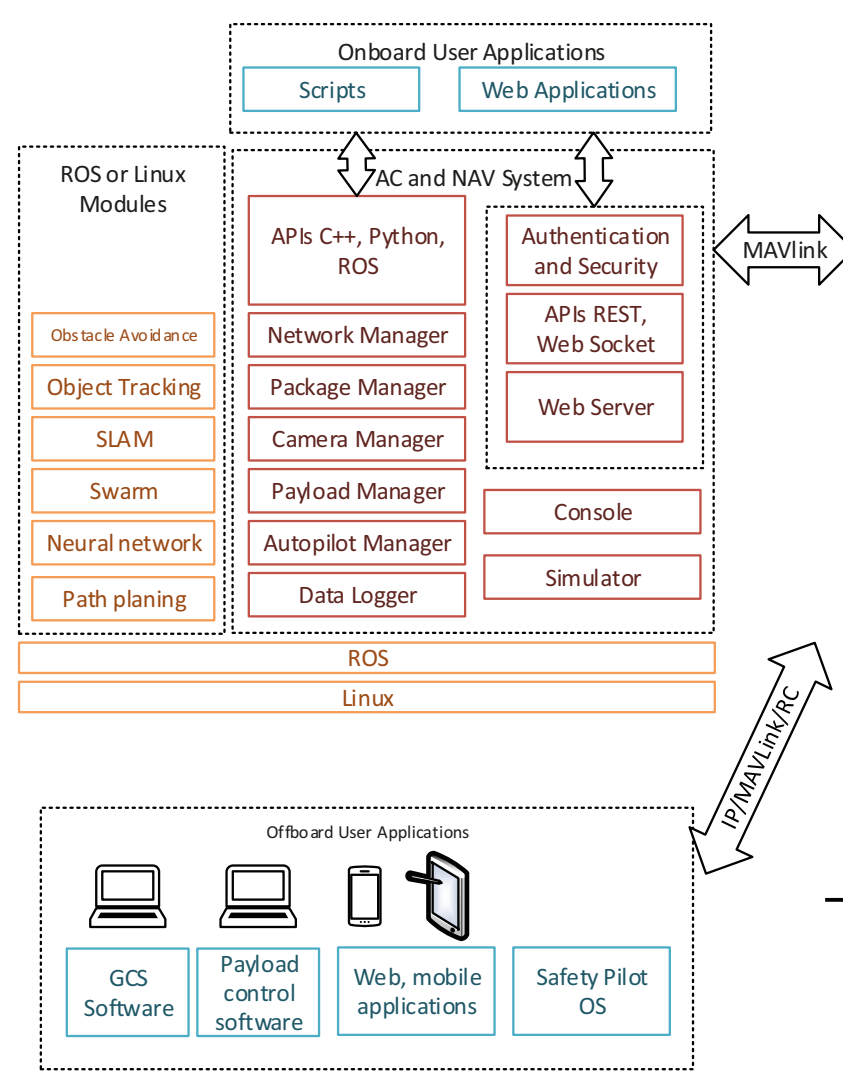

Fig. 18. Software architecture (acc. these authors)

\section{COMMUNICATION CHANNELS}

The communication and control link, in addition to the platform itself and the ground control station, is the most important module of the UVS and provides the necessary information exchange between the SCS and the proper platform systems. Communication with the USV is conducted in two directions : signals are sent towards the platform (uplink), and signals are received from the platform (downlink). The uplink consists of radio control signals for the payload and USV. The downlink provides telemetric data and sensors readings. In the presented application, the communication with the platform is conducted in radio bands and with transmission power that can be used without proper permission, i.e. in channels that have been allocated to the public (ISM Bands). According to the definition, the industrial, scientific and medical (ISM) radio bands are those intended to use radio frequency energy for industrial, scientific and medical purposes other than telecommunication. ISM bands allows to use transmitting devices without a radio license, within assigned frequencies and limited power. As a result, the frequency range and maximum power is strictly defined and manufacturers can build and market any radio devices that meet the conditions set out in the regulations, and users can use them without permission. In practice, this means that the released bands are very disrupted and there are many different radio devices working within the ISM bands.
For the presented project, three main communication channels have been chosen, prioritized and assigned in accordance with main function and range. The safety pilot (radio control) have the highest priority (should have the best range) and the lowest demands as for the channel width. The $433 \mathrm{MHz}$ band has been assigned for the radio control application, with proven range up to $65 \mathrm{~km}$. The RC (radio control) channel is only uplink. The telemetric data, uplink and downlink, are assigned to $868 \mathrm{MHz}$ band. Two longrange radio modems are assigned for the telemetric data exchange between platform and shore station. The telemetric data allows to control and monitor vessel parameters, current state and position. It allows to operate beyond line of sight, with proven range up to $60 \mathrm{~km}$. The most demanding channel, that for high-rate video data and payload control data , is assigned to $2,4 / 5,8 \mathrm{GHz}$ band. The channel allows to transfer a live video feed form all cameras mounted on board, fully control commuters on board and transfer all raw sensors data from sensor module. The range of the link is proved to be up to $6 \mathrm{~km}$. It should be mentioned, that payload is automatically controlled by the onboard commuter and its constant monitoring is not required. The camera live feed is not required during live operation over a long distance, while a unit operates on the open water. All anti-collision manoeuvres are carried out automatically, without operator attention taking for consideration international regulations. The problem of international regulations for autonomous surface vehicle was discussed in [18].

\section{CONCLUSIONS}

The universal autonomous control and management system for multipurpose unmanned surface vessel has been designed and implemented on the catamaran vessel tested and evaluated during the real environment trials on the lake (Fig.19).

The presented floating platform solution developed by the integrating of the unmanned vessel system with the optimizing of the platform drive and control and the use of real-time sensors constitutes a novelty not only in the Polish market, but also in a worldwide scale. 


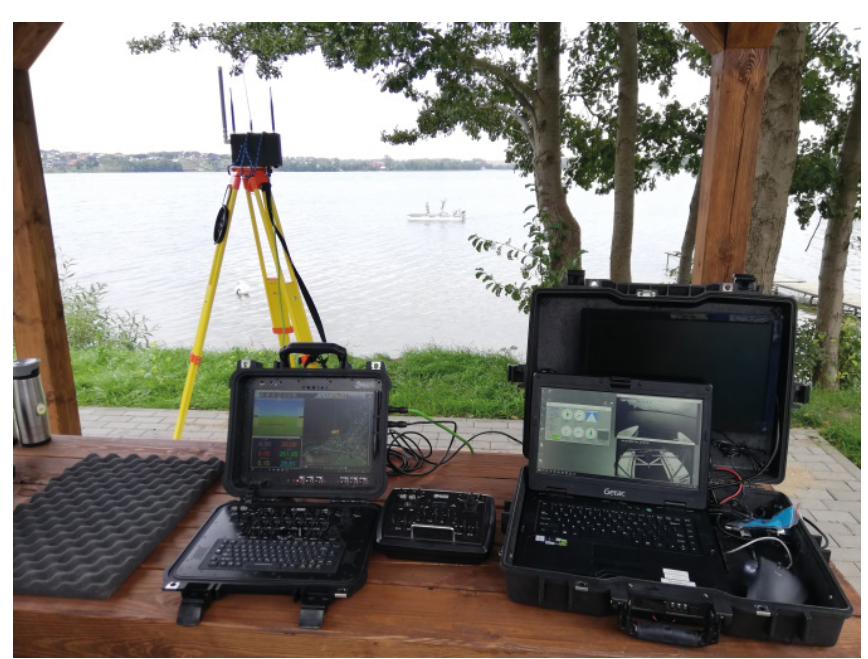

Fig. 19. Fully unmanned vessel system deployed for trials (first plane: shore station, safety pilot, hydrographic console; second plane: shore communication module; third plane: HydroDron unit afloat)

An important feature of the HydroDron is the ability to use it also for tasks other than hydrographic measurements, such as monitoring rivers, lakes and other water areas, environmental quality testing etc.

The possibility to replace sensors in modular kits and apply a universal control software designed for the use with different devices provides the ability to use a selected device in multiple variations.

\section{ACKNOWLEDGMENTS}

The presented work was financed from the European Regional Development Fund under the 2014-2020 Operational Programme Smart Growth. Project entitled „Developing of autonomous/remote operated surface platform dedicated to hydrographic measurements on restricted reservoirs" implemented as a part of the National Centre for Research and Development competition „INNOSBZ”.

\section{BIBLIOGRAPHY}

1. Barton A., Volna E.: Control of Autonomous Robot using Neural Networks. Proceedings of the International Conference on Numerical Analysis and Applied Mathematics 2016 (ICNAAM-2016), vol. 1863, Rhodes, Greece 2016.

2. Burdziakowski P., Szulwic J.: A commercial of the shelf components for a unmanned air vehicle photogrammetry, $16^{\text {th }}$ International Multidisciplinary Scientific GeoConference SGEM 2016, Albena, Bulgaria, 2016

3. Droeschel D., Schwarz M. Behnke S.: Continuous mapping and localization for autonomous navigation in rough terrain using a 3D laser scanner, Robotics and Autonomous Systems, vol. 88, pp. 104-115, 2017.
4. Guan R.P., Ristic B., Wang L.P. et al.: Feature-based robot navigation using a Doppler-azimuth radar, International Journal of Control, vol. 90, issue 4, pp. 888-900, 2017.

5. Guerrero J.A., Jaud M., Lenain R. et al.: Towards LIDARRADAR based Terrain Mapping, 2015 IEEE International Workshop on Advanced Robotics and its Social Impacts (ARSO), Lyon, France , 2015.

6. Guo W., Wang S., Dun W.: The Design of a Control System for an Unmanned Surface Vehicle, Open Autom. Control Syst. J., vol. 7, pp. 150-156, 2015.

7. Hollinger J., Kutscher B. Close B.: Fusion of Lidar and Radar for detection of partially obscured objects. Unmanned Systems Technology XVII, Baltimore, MD, vol. 9468 , 2015.

8. Huang L., Chen S., Zhang J. et al.: Real-Time Motion Tracking for Indoor Moving Sphere Objects with a LiDAR Sensor, Sensors, vol. 17, issue 9, 2017.

9. Jeon H.C., Park Y.B., Park C.G.: Robust Performance of Terrain Referenced Navigation Using Flash Lidar, Proceedings of the 2016 IEEE/Ion Position, Location and Navigation Symposium (PLANS), pp. 970-975, Savannah, GA, 2016

10. Jiang Z., Wang J., Song Q.et al.: Off-road obstacle sensing using synthetic aperture radar interferometry, Journal of Applied Remote Sensing, vol. 11, 2017.

11. Jo J., Tsunoda Y., Stantic B. et al.: A Likelihood-Based Data Fusion Model for the Integration of Multiple Sensor Data: A Case Study with Vision and Lidar Sensors. Robot Intelligence Technology And Applications 4, vol. 447, pp. 489-500, 2017.

12. Jooho L., Joohyun W., Nakwan K.: Obstacle Avoidance and Target Search of an Autonomous Surface Vehicle for 2016 Maritime RobotX Challenge. IEEE OES International Symposium on Underwater Technology (UT), Busan, South Korea , 2017.

13. Kazimierski, W., Stateczny, A.: Fusion of Data from AIS and Tracking Radar for the Needs of ECDIS. Book Group Author(s): IEEE Conference: Signal Processing Symposium (SPS), Jachranka, Poland , 2013.

14. Ko B., Choi H.J., Hong C. et al.: Neural Network-based Autonomous Navigation for a Homecare Mobile Robot. 2017 IEEE International Conference On Big Data And Smart Computing (BIGCOMP), pp. 403-406, Jeju, South Korea , 2017.

15. Lil J., Bao H., Han X. et al.: Real-time self-driving car navigation and obstacle avoidance using mobile $3 D$ laser 
scanner and GNSS. Multimedia Tools and Applications, vol. 76, pp. 23017-23039, part B, 2016.

16. Lisowski J: Optimization-supported decision-making in the marine game environment. Mechatronic Systems, Mechanics And Materials II, Book Series: Solid State Phenomena, vol. 210, pp. 215-222, 2014.

17. Lisowski J: The optimal and safe ship trajectories for different forms of neural state constraints. Mechanics and Materials II, Book Series: Solid State Phenomena, vol.180, pp. 64-69 , 2012.

18. Mei J.H., Arshad M.R.: COLREGs Based Navigation of Riverine Autonomous Surface Vehicle. IEEE 6TH International Conference on Underwater System Technology, pp.145-149, Malaysia, 2016.

19. Mikhail M., Carmack N.: Navigation Software System Development for a Mobile Robot to Avoid Obstacles in a Dynamic Environment using Laser Sensor, SOUTHEASTCON 2017, Charlotte, NC, 2017.

20. Praczyk T.: Neural anti-collision system for Autonomous Surface Vehicle, Neurocomputing, vol. 149, pp. 559-572 , 2015.

21. Specht C, Weintrit A., Specht M.: Determination of the territorial sea baseline - Aspect of Using Unmanned Hydrographic Vessels, Transnav-International Journal on Marine Navigation and Safety of Sea Transportation, vol. 10, pp. 649-654, 2016.

22. Specht C. Switalski E., Specht M.: Application of an Autonomous /Unmanned Survey Vessel (ASV/USV) in bathymetric measurements, Polish Maritime Research, No. 24, pp.36-44, 2017.

23. Stateczny A., Gronska D., Motyl W.: Hydrodron - New Step for Professional Hydrography for Restricted Waters, 2018 Baltic Geodetic Congress (BGC Geomatics), pp. 226-230, Olsztyn, Poland, 2018.

24. Williams G.M.: Optimization of eye-safe avalanche photodiode lidar for automobile safety and autonomous navigation systems, Optical Engineering, vol. 56, issue 3, 2017.

\section{CONTACT WITH THE AUTHORS}

\author{
Andrzej Stateczny \\ e-mail: a.stateczny@marinetechnology.pl \\ Marine Technology Ltd. \\ Cyfrowa 6, B.3.04a \\ 71-441 Szczecin \\ POLAND \\ Pawel Burdziakowski \\ e-mail:pawel.burdziakowski@pg.edu.pl \\ Gdansk University of Technology \\ Faculty of Civil and Environmental Engineering \\ Narutowicza 11/12 \\ 80-233 Gdansk \\ Poland
}

